\title{
The Effectiveness of the Cold Therapy (cryotherapy) in the Management of Inflammatory Parameters after Removal of Mandibular Third Molars: A Meta-Analysis
}

\author{
Ighor Andrade Fernandes ${ }^{1}$ Anna Catharina Vieira Armond $^{1} \quad$ Saulo Gabriel Moreira Falci ${ }^{1}$ \\ ${ }^{1}$ Section of Oral and Maxillofacial Surgery, Department of Dentistry, \\ Universidade Federal dos Vales do Jequitinhonha e Mucuri (UFVJM), \\ Diamantina, MG, Brazil \\ Int Arch Otorhinolaryngol 2019;23:221-228. \\ Address for correspondence Ighor Andrade Fernandes, MSc, Setor de \\ Cirurgia Oral e Maxilofacial, Departamento de Odontologia, \\ Universidade Federal dos Vales do Jequitinhonha e Mucuri (UFVJM), \\ Rua da Glória, 187, Diamantina, 39100-000, MG, Brazil \\ (e-mail: ighor.af@gmail.com).
}

\begin{abstract}
Introduction Cold therapy (cryotherapy) is a common procedure recommended by dental surgeons after surgical removal of third molars, which is an invasive intervention that often deals with an expressive inflammatory response.

Objective To investigate whether cryotherapy interferes with clinical outcomes such as pain, edema, and trismus in the postoperative period of mandibular third molar surgeries. Data Synthesis An electronic search was conducted in the OVID, PubMed, VHL, Science Direct, Cochrane Library, and Web of Science databases, through March 2018. The eligibility criteria included clinical trials that evaluated the effect of cryotherapy in at least one of the following variables: pain, swelling, and trismus.

Two independent reviewers assessed the studies. The methodological quality of each article was analyzed. The search strategy resulted in 1,088 articles. Following the selection process, 11 studies were included in the systematic review and 4 of them in the meta-analysis. High risk of bias was found in most of the studies according to the Cochrane Handbook assessment. Patients receiving cryotherapy had less edema than patients in the control group at second day follow-up (mean difference [MD]: -0.94; $95 \% \mathrm{Cl}[-1.49 ;-0.39])$. There were no statistically significant results when comparing

Keywords

- third molar

- cryotherapy

- cold therapy

- maxillofacial surgery trismus between experimental and control group (MD: $0.43 ; 95 \% \mathrm{Cl}[-0.34 ; 1.20]$ ). There were insufficient available data to support influences in postoperative pain.

Conclusions Cryotherapy applied on the first day after mandibular third molar removal can manage edema in the patients. Well-designed randomized clinical trials to test the efficacy of cryotherapy after surgical removal of third molars are needed to justify its indication.
\end{abstract}

\section{Introduction}

The removal of mandibular third molars is one of the most common procedures performed by dental surgeons, either for prophylactic purposes or to treat problems caused by the presence of these teeth, such as caries in adjacent teeth, ${ }^{1}$ periodontal changes, ${ }^{2}$ or lack of space in the dental arch and

(1) Ighor Andrade Fernandes's ORCID is https://orcid.org/00000002-3767-5197.

received

April 11, 2018

accepted

December 2, 2018

published online

March 13, 2019 consequent problems in dental occlusion. ${ }^{3}$ However, even with the best preoperative procedures and trans-surgical ducts, exaggerated inflammatory reactions that cause trismus, edema, and pain may be present. ${ }^{4}$ Despite these signs and symptoms, which are expected due to the inflammatory response to the tissue trauma resulting from the surgery, they often cause discomfort to the patient and can affect them in daily activities. ${ }^{5-7}$

The cold therapy, or cryotherapy, is the local or systemic application of cold through various methods for therapeutic

Copyright (@) 2019 by Thieme Revinter Publicações Ltda, Rio de Janeiro, Brazil

\section{License terms}

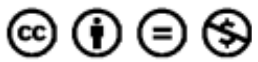


purposes to lower the temperature of the skin and subcutaneous tissues. ${ }^{8}$ Physicians often recommend the use of ice for patients who have undergone physical injuries and surgical procedures with therapeutic purposes, since this technique is known to control inflammation and hemorrhage due to vasoconstriction mediated by local cooling. ${ }^{9-12}$

In the literature, we can find some studies that deal with the efficiency of cryotherapy in the control of inflammatory reactions after third molar removal surgeries.,9,13-16 Based on the results of van der Westhuijzen et al, ${ }^{17}$ the use of ice for 24 hours after third molar surgery was not effective in reducing pain and edema in an observational study with 60 patients. However, according to Laureano Filho et al, ${ }^{16}$ and other authors, $4,9,13-15,18$ ice compression effectively helps reduce pain and edema after third molar extraction. Thus, the information on the use of cryotherapy after third molar removal is still limited and controversial. ${ }^{13}$

The aim of this study was to compare, through a scientifically based approach, whether cryotherapy (cold therapy) interferes with clinical outcomes such as pain, edema, and trismus in the postoperative period of third molar surgeries.

\section{Review of the Literature}

This study was conducted according to the Preferred Reporting Items for Systematic Reviews and Meta-Analyses (PRISMA) statement and the Cochrane Handbook (Higgins \& Green 2011). ${ }^{19}$

The question to be answered was created using the PICO process (that is, $\mathrm{P}=$ patient, problem, or population, $\mathrm{I}=$ intervention, $\mathrm{C}=$ comparison, and $\mathrm{O}=$ outcomes), as follows: the population included patients undergoing surgical removal of mandibular third molars; the intervention was the use of cryotherapy; the comparison was the non-use of cryotherapy; the outcome was reduced inflammatory parameters after a surgical removal of mandibular third molars. Therefore, the study aims to answer the following question: is there significant effect of using cryotherapy on the inflammatory parameters after a surgical removal of mandibular third molars?

\section{Eligibility}

The inclusion criteria for this systematic review were: clinical trials, randomized or not, analyzing the use of cryotherapy, or cold therapy, to determine the incidence of clinical parameters following surgical removal of mandibular third molars. The papers must have to evaluate at least one of the variables to be included: pain, swelling, and trismus.

The reviewer's examination of full texts led to the exclusion of articles that were case reports, technical notes, event summaries, animal studies, in vitro studies, literature reviews, and studies that did not evaluate the efficacy of cold therapy on the effects of postoperative inflammation after mandibular third molars extraction operations.

\section{Search Strategy}

The electronic search was conducted without language restriction in the OVID, PubMed, Virtual Health Library (VHL), Science Direct, Cochrane Library, and Web of Science, and included publications through March, 2018. Combinations of medical subject heading (MeSH) terms were used to find the maximum number of possible studies available in the literature. The following terms were used in the databases: "third molar AND surgery AND ice" and "third molar AND surgery AND cryotherapy." Furthermore, a manual search for relevant studies was performed in the thesis bank and in references of included articles.

After the search was concluded, two reviewers (I. A. F. and A. C. V. A.) independently first examined paper titles and abstracts, after removing all duplicates, selecting papers for full-text screening that could potentially meet the inclusion criteria for this systematic review. After independent reading, the reviewers compared their results, disagreements between them were resolved by discussion and a third reviewer (S. G. M. F.) was consulted in case of persistent disagreements for a final decision of the studies that would pass to the full text assessment. At this point, the reviewer's examination of full texts led to the predetermined criteria. Papers fulfilling all selection criteria were processed for data extraction.

The quality of included papers was assessed by the reviewers (I. A. F. and A. C. V. A.) using the Cochrane Handbook for Systematic Reviews of Interventions 5.0.1 (Higgins \& Green 2011). The classification of the potential risk of bias for each study was based on the following criteria: randomized sequence generation; allocation sequence concealment; blinding of participants, personnel, and outcome assessors; incomplete outcome data; selective outcome reporting; and certainty of the application of the cryotherapy at the adequate moments. Each study was submitted to analysis, and the criteria were rated as exhibiting low, high, or unclear (no information or uncertain) risk of bias. The disagreements between reviewers were resolved by consensus for the final classification.

The data were collected from eligible articles and the studies characteristics are expressed in -Table 1. Mean values and standard deviations were extracted, when it was available. The results were checked among reviewers after data collection to ensure accuracy.

\section{Statistical Analysis}

The meta-analysis was performed using the software " $R$, version 3.3.1" (R Foundation for Statistical Computing). The meta and metafor packages were used to conduct the statistical calculation and the forest plots. The heterogeneity between the results of the studies was assessed using the $\mathrm{I}^{2}$ statistical test. This test expresses the percentage of the variation across studies. Values above 25 and $50 \%$ were considered an indicative of moderate and high heterogeneity, respectively. As heterogeneity was present $\left(\mathrm{I}^{2}>0\right)$ in all tests, the random effect model was used to perform the metaanalysis. $^{20}$

\section{Results of Systematic Search}

The electronic literature search resulted in the identification of 1,080 studies (Cochrane 15 articles, OVID 249 articles, PubMed 29 articles, Science Direct 752 articles, VHL 23 articles, and Web of Science 16 articles). The search in the 


\begin{tabular}{|c|c|c|c|c|c|c|c|c|c|c|c|}
\hline 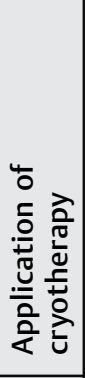 & 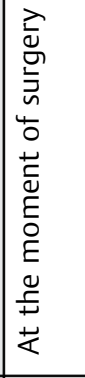 & 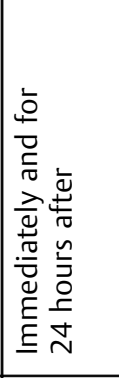 & 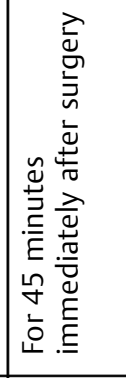 & 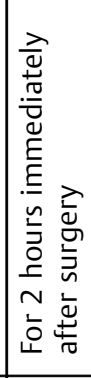 & 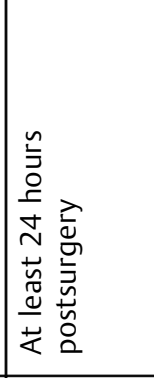 & 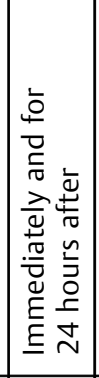 & 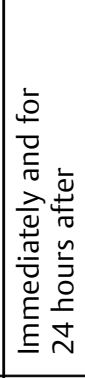 & 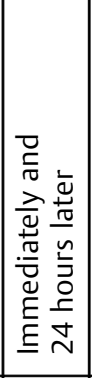 & 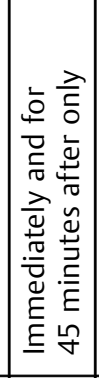 & 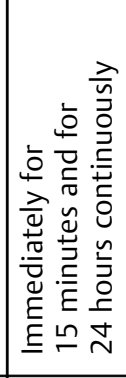 & 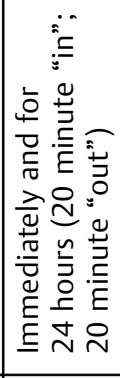 \\
\hline 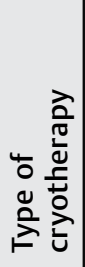 & 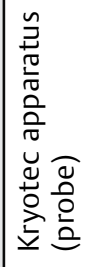 & 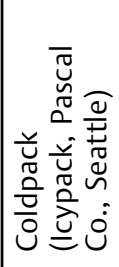 & 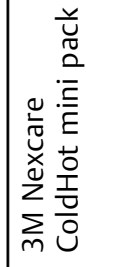 & 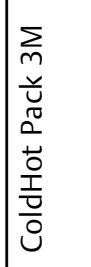 & 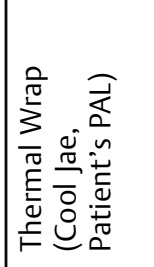 & \begin{tabular}{|l}
$\underline{u}$ \\
$\tilde{O}$ \\
$\underline{\Xi}$ \\
$\underline{\Delta}$
\end{tabular} & 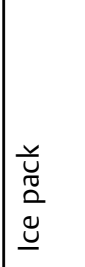 & 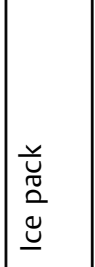 & 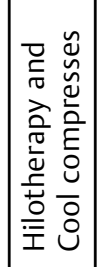 & 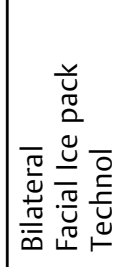 & $\begin{array}{l}\underline{\ddot{u}} \\
\tilde{D} \\
\underline{\tilde{U}}\end{array}$ \\
\hline 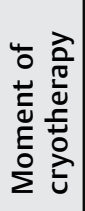 & 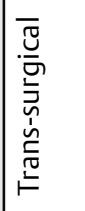 & 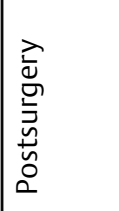 & 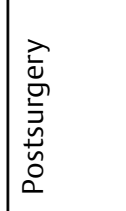 & 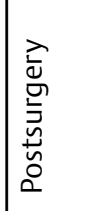 & 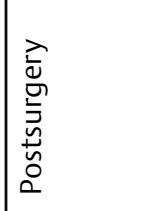 & 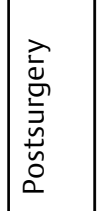 & 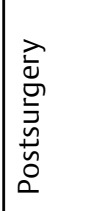 & 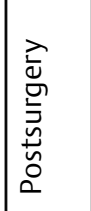 & 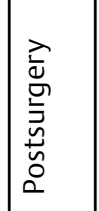 & 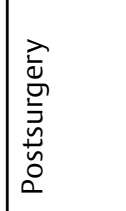 & 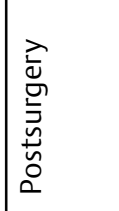 \\
\hline $\begin{array}{l}\frac{0}{7} \\
3^{1} \\
\frac{0}{0} \\
4\end{array}$ & 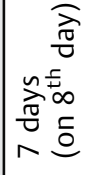 & 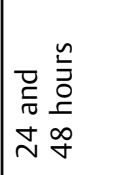 & 离 & $\frac{n}{\underset{\pi}{0}}$ & $\begin{array}{l}\stackrel{n}{\sigma} \\
\underset{\sigma}{\sigma} \\
\nabla \\
\tau\end{array}$ & 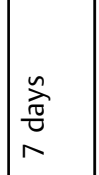 & $\begin{array}{l}\stackrel{n}{\pi} \\
\frac{\pi}{\sigma} \\
r\end{array}$ & $\begin{array}{l}\frac{n}{\sqrt{\sigma}} \\
\frac{\pi}{r} \\
r\end{array}$ & 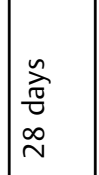 & 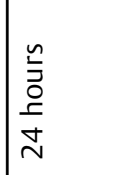 & $\begin{array}{l}\frac{n}{\pi} \\
\frac{\pi}{\sigma} \\
r\end{array}$ \\
\hline$\frac{\mathbb{E}}{\sum}$ & 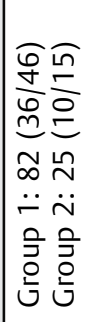 & F & 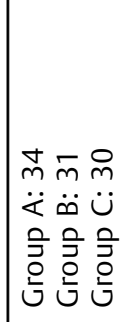 & $\mid \begin{array}{l}\frac{a}{2} \\
\frac{1}{0} \\
\stackrel{d}{d} \\
\qquad \frac{8}{\gamma}\end{array}$ & 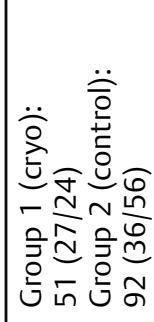 & 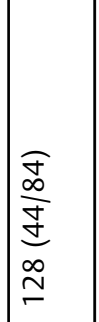 & 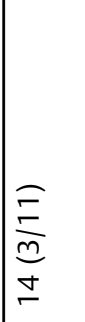 & 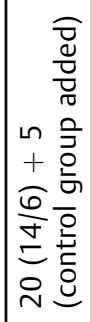 & 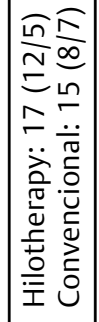 & 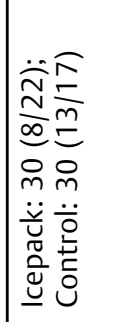 & $\mid \begin{array}{l}\frac{N}{N} \\
\stackrel{N}{\Xi} \\
0 \\
m\end{array}$ \\
\hline 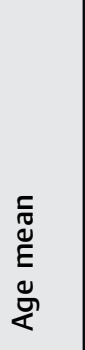 & 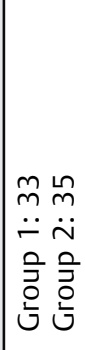 & 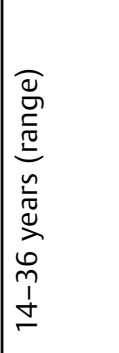 & 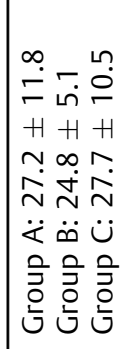 & 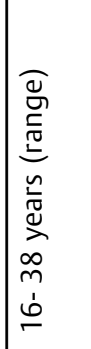 & 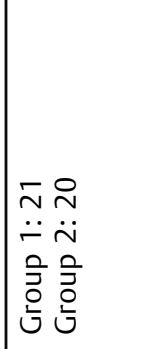 & 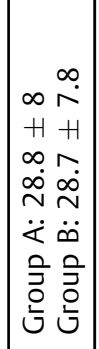 & $\stackrel{\searrow}{\sim}$ & $\stackrel{\Perp}{\sim}$ & 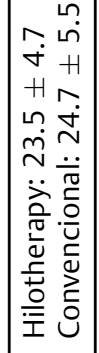 & 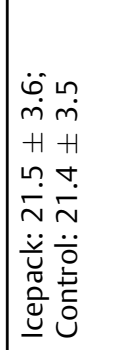 & $\begin{array}{l}\tilde{i} \\
+1 \\
\sim \\
\tilde{N}\end{array}$ \\
\hline 站 & 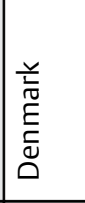 & 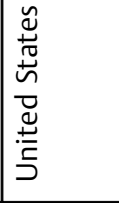 & 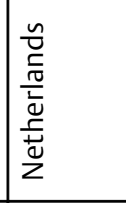 & 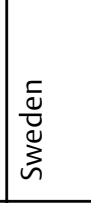 & 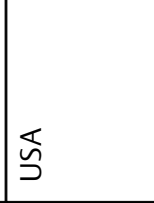 & 蒙 & \begin{tabular}{|l}
$\overline{\bar{N}}$ \\
$\stackrel{\mathbb{N}}{0}$ \\
\end{tabular} & 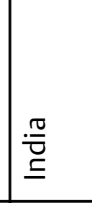 & 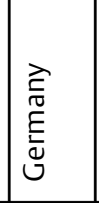 & 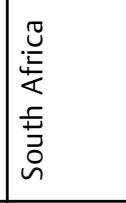 & 듄 \\
\hline 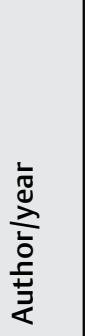 & 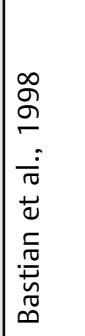 & 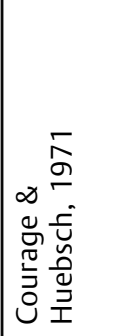 & 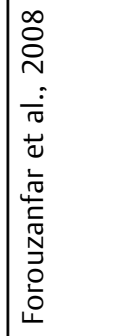 & 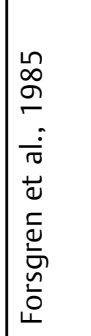 & 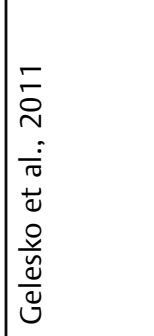 & 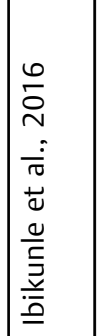 & 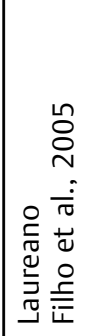 & 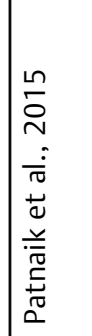 & 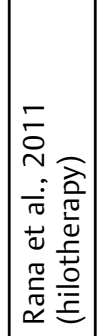 & 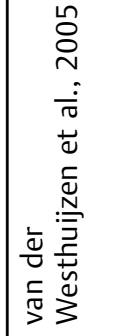 & 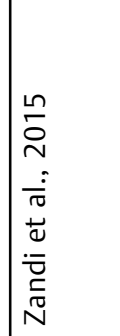 \\
\hline
\end{tabular}


reference lists of selected papers and hand search revealed one additional relevant paper. ${ }^{21}$ From the total, 175 duplicates were removed, resulting in 906 articles for analysis. After 2 independent authors screened titles and abstracts, 887 studies were excluded for not fulfilling the inclusion criteria. At this stage, the reviewers retrieved 19 studies for full-text assessment. Eight articles were excluded after fulltext reading. A total of 11 studies $^{4,13-17,21-25}$ were included in the qualitative synthesis, and, from these, 4 stu$\operatorname{dies}^{4,16,21,24}$ were included in the meta-analysis. The flowchart of papers selection and the reasons for exclusion are summarized in -Fig. 1.

\section{Description of the Studies}

The relevant characteristics of the included papers are presented in -Table 1. Follow-up periods ranged from 1 to 28 days. Among the 11 studies included in the qualitative synthesis, just $1^{22}$ used a probe (Kryotec) to apply cryotherapy method in the trans-surgical moment, the others used types of icepacks in the postsurgery period, highlighting 1 study, ${ }^{14}$ which compared hilotherapy and cool compressions. In relation to the design of the study, six stu$\operatorname{dies}^{13-15,17,21,25}$ did the procedures without using the split mouth method; they extracted just one third molar or extracted all of them at the same moment. The variable pain was assessed in all studies, but the following ones could also be found: swelling, discomfort sensation, duration of the surgery, infection, trismus, body temperature, wound healing, and quality of life.

The highest average age was 35 years, ${ }^{22}$ while the lowest average age was 20 years. $^{25}$ The publication year of the studies ranged from $1971^{23}$ to $2016 .^{15}$

Investigators, in some studies, have found that cryotherapy can reduce pain, ${ }^{25}$ edema, ${ }^{21}$ and has a low frequency of complications for the patients after wisdom tooth removal. ${ }^{16,22}$ Courage et $\mathrm{al}^{23}$ concluded that cryotherapy deserves to be an approach for the oral surgeon, since it can reduce facial swelling and alleviate pain. One study compared cold compressions with compression alone and found that the effect in both treatment modalities is equal in reducing pain after oral surgery. ${ }^{13}$ Some studies $4,17,24$ showed that postoperative external application of cold dressings does not diminish swelling, trismus or pain after removal of impacted wisdom tooth. One study ${ }^{15}$ suggested that the quality of life of subjects undergoing third molar surgery was much better in those who had cryotherapy compared with those who did not have it. Another included study $^{14}$ concluded that hilotherapy is more effective in managing swelling and pain than cryotherapy after wisdom tooth removal.
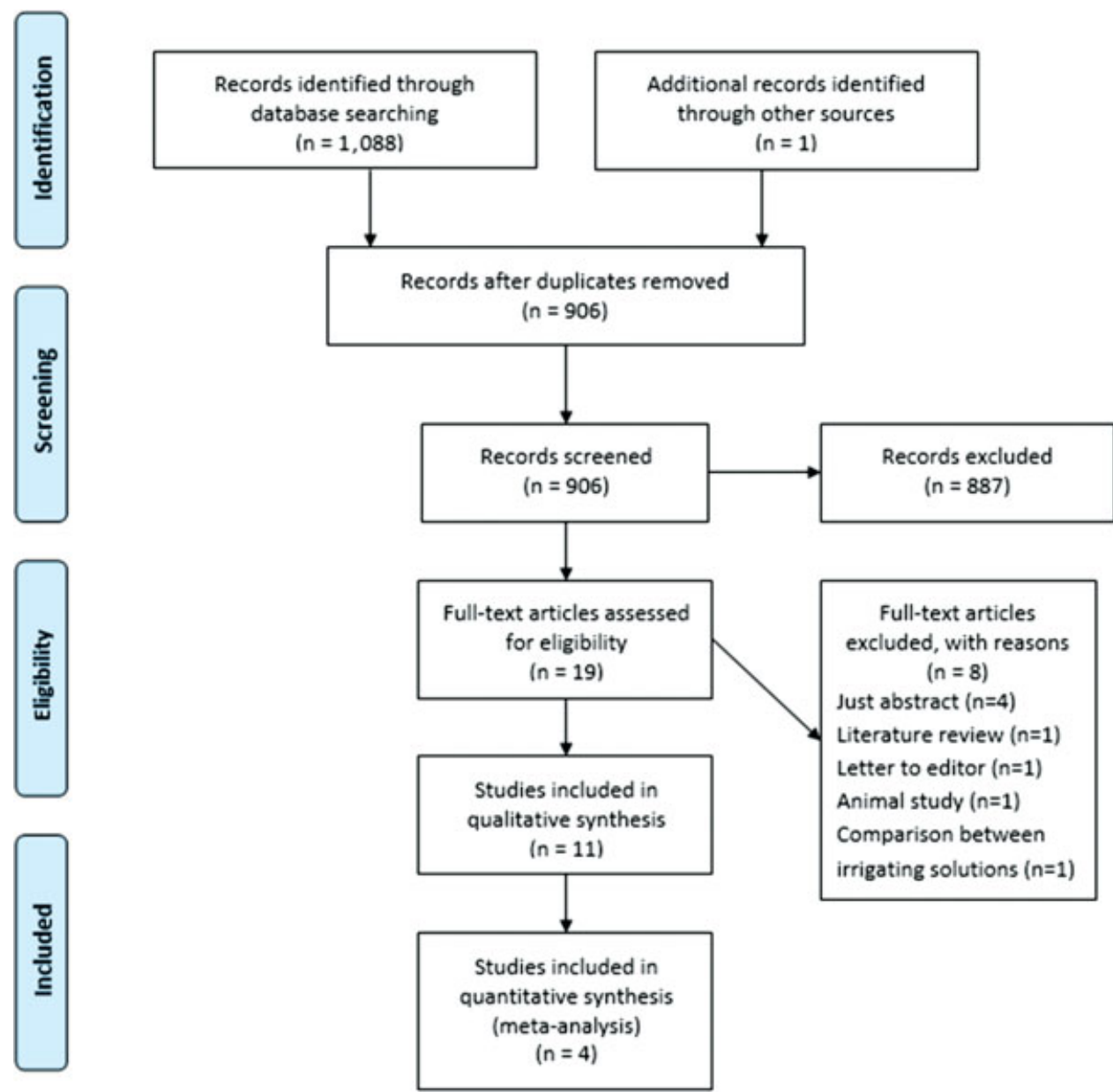

Fig. 1 Flow diagram showing the article selection process. 


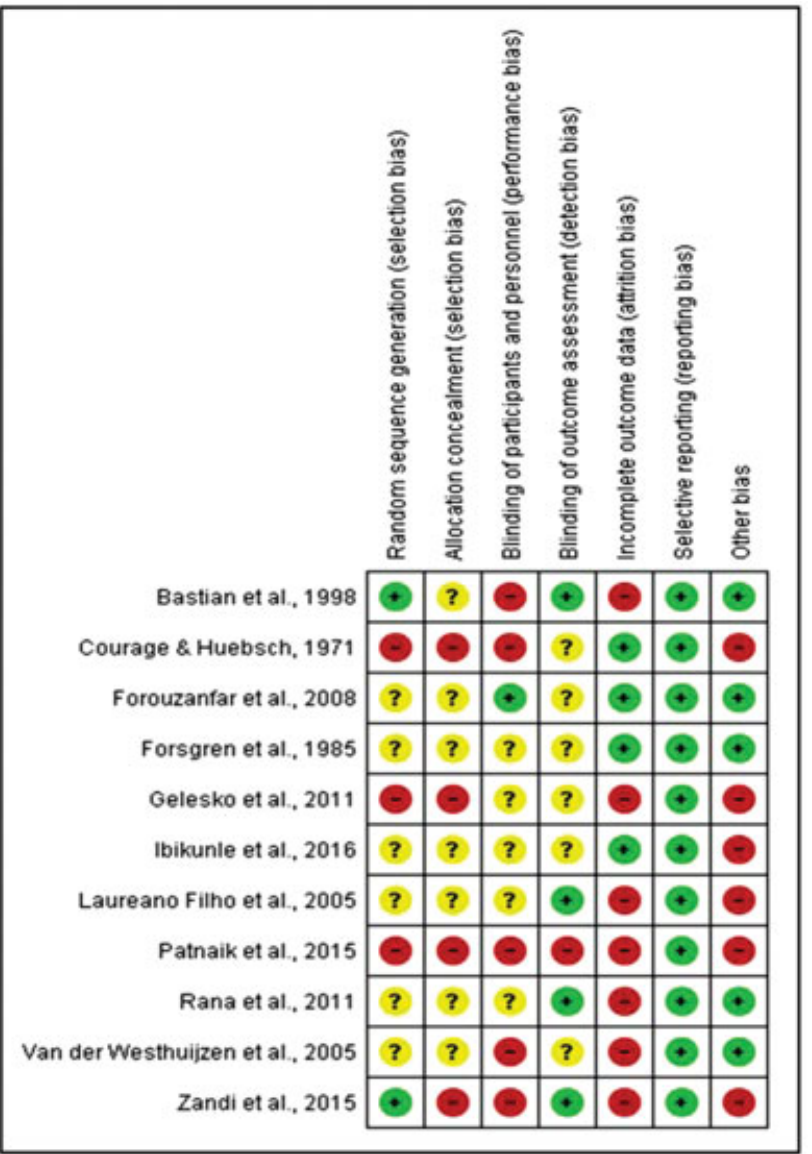

Fig. 2 Quality assessment of the articles included in the review.

\section{Quality Assessment}

The results of the quality assessment of the included studies are presented in - Fig. 2. Risk of bias was high in most of the criteria categories in many studies. The perspective of the certainty of the application of cryotherapy at the adequate moments was assessed, once we considered this aspect an important parameter to verify the real efficiency of the cryotherapy in the relief of inflammatory responses after surgical removal of mandibular third molars.

\section{Meta-analysis}

Meta-analysis comparing the effect of cryotherapy versus control group in swelling and trismus could be performed; the other clinical parameters could not be included in the quantitative synthesis due to the absence of standardized methodology.

The forest plot for the effect of the intervention in the postoperative edema is shown in - Fig. 3. The meta-analysis for edema included a total of 85 participants ( 50 in the experimental group and 35 in the control group). After data extraction, we used two of the selected studies for metaanalysis. The results showed that on the $2^{\text {nd }}$ day of follow-up, participants receiving cryotherapy had less swelling than did participants in the control group (mean difference [MD]: $-0.94 ; 95 \%$ CI $[-1.49 ;-0.39])$. However, on the $7^{\text {th }}$ day of follow-up (MD: - 0.35 ; 95\%CI [-1.34; 0.64]) and when evaluating the overall results of efficiency of cryotherapy (MD: -0.53 ; 95\%CI $[-1.23 ; 0.17])$, there was no statistically significant difference between the control and the experimental groups.

The forest plot for the effect of the cryotherapy in the postoperative trismus is shown in - Fig. 4. The meta-analysis for trismus included a total of 203 participants (109 in the experimental group and 94 in the control group). Four of the 11 selected studies were used for this meta-analysis after data extraction. The results showed that on the $1^{\text {st }}$ (MD: 0.55; $95 \% \mathrm{CI}[-2.14 ; 3.24]), 2^{\text {nd }}(\mathrm{MD}: 2.37 ; 95 \% \mathrm{Cl}[-0.50 ; 5.24])$, and $7^{\text {th }}$ (MD: $0.76 ; 95 \% \mathrm{CI}[-3.10 ; 4.63]$ ) days of follow-up, there was no statistically significant difference between control group and experimental group. The overall result (MD: 0.43; $95 \% \mathrm{CI}[-0.34 ; 1.20])$ just confirmed the insignificant efficacy of cryotherapy in reducing trismus after third molar removal.

\section{Discussion}

The relief of postoperative discomfort due to the inflammatory process after extraction of mandibular third molars is a concern that most affects the patients and the surgeons. Cold

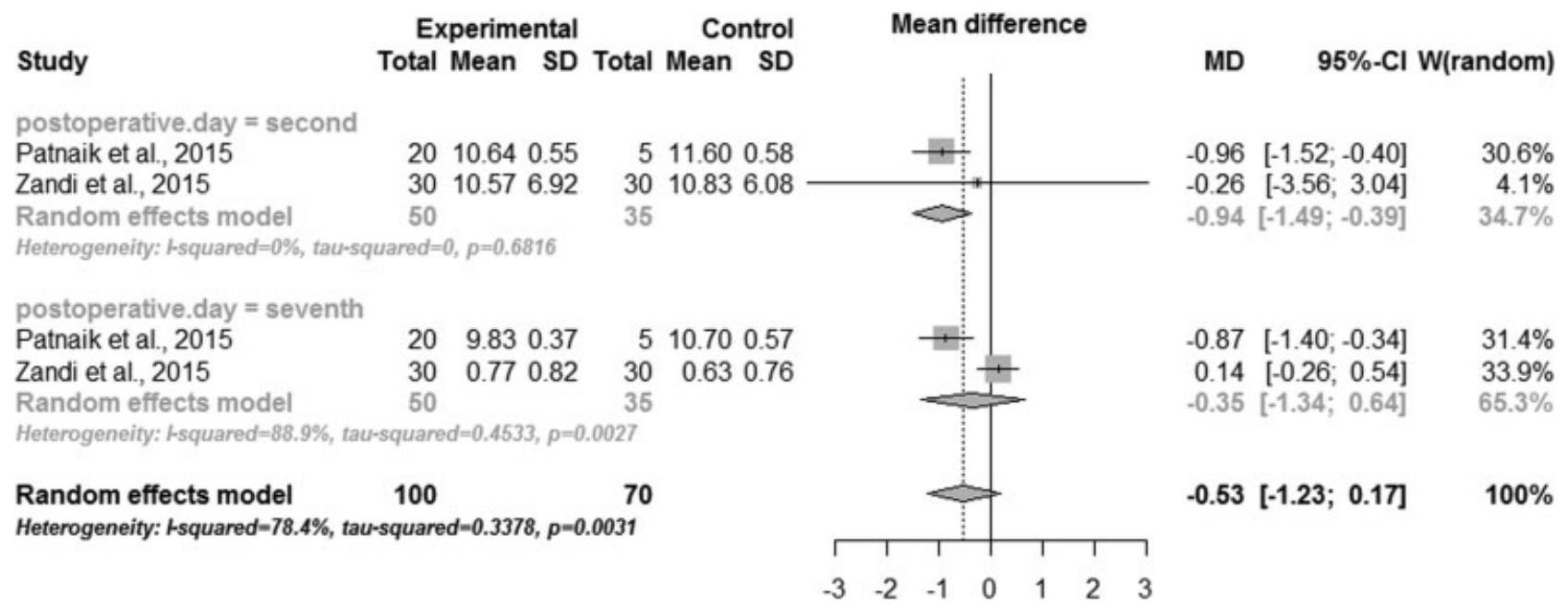

Fig. 3 Forest plot for the effect of the cryotherapy in the postoperative edema. Abbreviations: $95 \% \mathrm{Cl}, 95 \%$ confidence interval; $\mathrm{MD}$, Mean Difference; SD, standard deviation; W, Weight. 


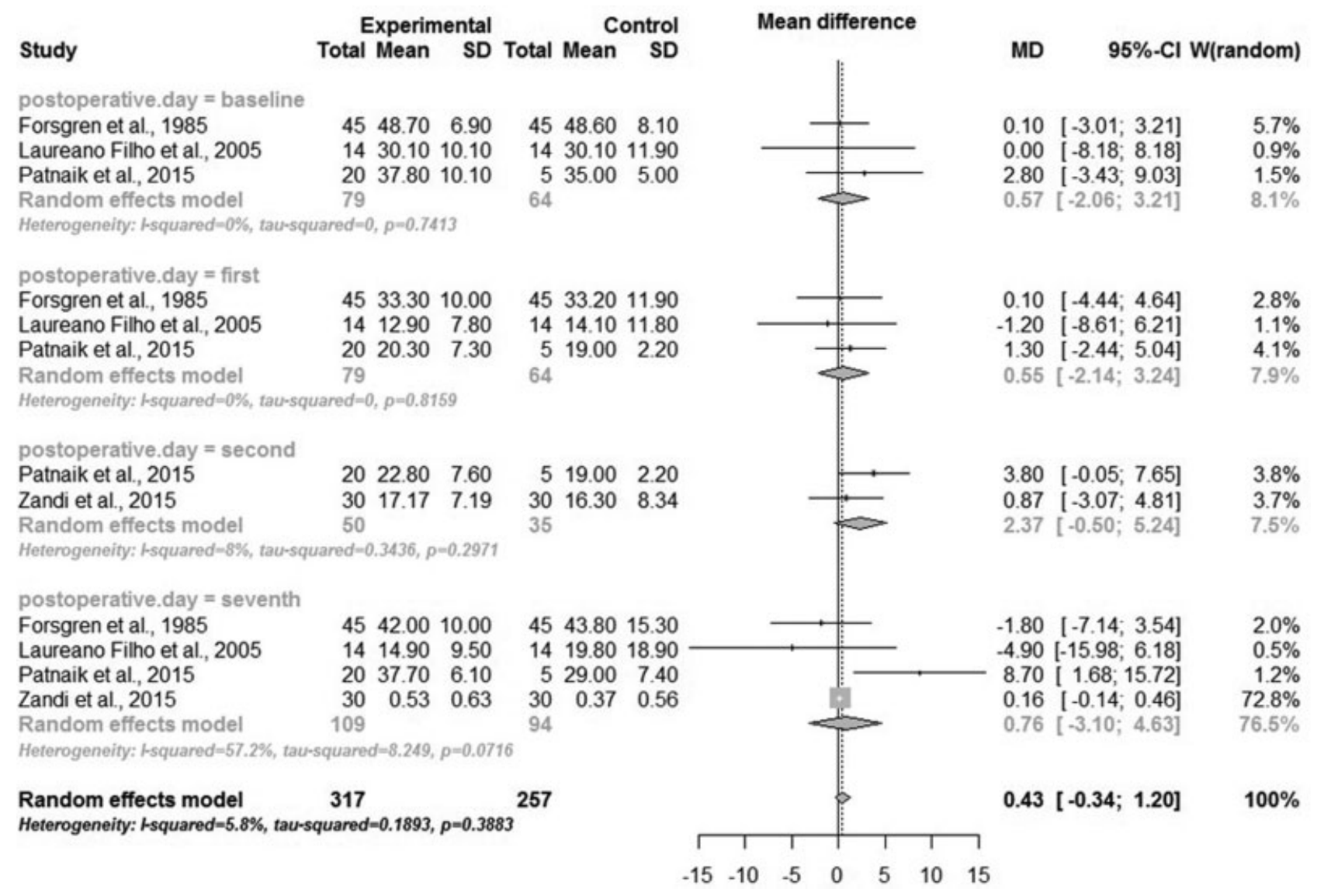

Fig. 4 Forest plot for the effect of the cryotherapy in the postoperative trismus. Abbreviations: $95 \% \mathrm{Cl}, 95 \%$ confidence interval; MD, Mean Difference; SD, standard deviation; W, Weight.

therapy is constantly used as a primary care after facial injuries. Although it is widespread, the available scientific data are still limited to support the real efficacy of this therapy. This procedure was originally applied due to the belief that the hot hyperemic area caused by the local inflammation was harmful, and that area must be cooled $^{26}$ to diminish the possible sequelae and discomfort. There are controversies regarding the therapeutic use of cold, even though it is one of the oldest practices after soft-tissue surgeries, first documented by Hippocrates (460-377 $\mathrm{BC}),{ }^{27}$ and that is why it is an important topic to discuss.

In a recent literature review undertaken in $2011,{ }^{28}$ it was observed that although ice therapy is a cheap, simple, and safe approach after oral surgeries, this practice is contraindicated in cold hypersensitive and intolerant patients, or in areas with circulatory, vascular, and regenerating nerves problems. ${ }^{29}$ Besides that, it was shown that no single modality of postoperative approach is effective in preventing complications without side effects. However, this previous review did not make a systematic search of relevant studies in the literature. The present systematic review aimed to provide updated scientific evidences and give a quantitative analysis of the effect of cryotherapy in clinical parameters after third molar surgeries.

As the results of the studies found in the literature show, it is still hard to allege the real effectiveness of cryotherapy. Some authors ${ }^{4,17,24}$ have found an insignificant association between better results on pain, edema, and trismus and the application of cold after third molar removal. The present meta-analysis confirms this fact in part, since it was not possible to perform quantitative analysis on pain parameters. It probably happened because of a non-standardized evaluation method for pain or just because the mean and standard deviation values were not available. However, it is known that when the local temperature falls below $14^{\circ} \mathrm{C}$, the transmission of nerve impulses along sensory and autonomic nerves is blocked, what is called "cold-induced neuropraxia", ${ }^{0}$ which causes local vasodilatation and paresthesia, resulting in reduced painful symptomatology. This fact can confirm the positive association between cryotherapy and relief of pain reported as results in some studies $^{15,16,22,23,25}$ of the present review.

Based on the meta-analysis for edema and trismus, it was possible to realize some important points in the physiology of cold application. On the $2^{\text {nd }}$ day of follow-up, the swelling was significantly reduced in the cryotherapy group than in the control group, and on the posterior follow-up periods this significance was not encountered. This could be explained by the fact that cooling causes an autonomic-mediated vasoconstrictive effect that reduces the edema formation. ${ }^{31,32}$ The lower the temperature, the slower will be the activity of neutrophils that mediate proinflammatory inducers and cytokines, which determines the early local fluid accumulation, and consequent swelling. It can confirm the results found in 
Bastian et al, Laureano Filho et al, Courage et al, and Patnaik et al, that show a significant efficacy of cryotherapy on postoperative swelling in third molar surgeries.

The trismus, evaluated in most of the included studies, ${ }^{4,14-17,21,24}$ did not present any significant different resolution over the studies when the patients treated with cryotherapy were compared with those in the control group. This must be a considerable result, since all included studies that evaluated trismus used the same reference to measure this parameter: the patient's maximum mouth opening from the maxillary incisive edge to the mandibular incisive edge.

Regarding the methodology for cold application, there was a variation among the studies. One author ${ }^{22}$ used a probe to apply cold in the surgical area and showed positive results in pain and edema. Another author ${ }^{25}$ used a thermal wrap, which had a positive influence in the highest pain levels experienced by patients undergone third molar surgeries. The other authors $^{4,13-17,21,23,24}$ used icepacks, or cold packs, to apply cryotherapy. The results among these studies that used cold packs were varied; some had significant results for cryotherapy and others did not. Thus, it could justify that the method for cryotherapy application may not influences the results. Even so, the variety of types of cryotherapy may be a limitation of this review, which suggests that a standardized method used in a large sample of patients in future studies could help investigators to verify the real efficacy of treatments.

More important than the type of cryotherapy used, the certainty of applying cryotherapy is crucial to proving effectiveness. From the 11 included studies, just $5^{13,14,17,22,24}$ reported that the researchers were concerned with verifying the correct and regular use of cold application by the patients according to the predefined methodology. It is another limitation of the present review, since the confirmation of the real effectiveness of cryotherapy could be changed if all studies wanted to be certain of the cold application, whether through a diary filled by the patients or through monitored application at the dental clinic.

It is interesting to note the comparison made by one study. Rana et al concluded in their study that hilotherapy is more effective than the simple application of cold therapy in managing edema and pain after third molar removal. It is explained by the differences in surface contact, with the hilotherapy covering a wider area when applied on the face. Other reasons are the variable temperature of the standard cryotherapy, which can become warmer over the time, and the influence of some materials used to wrap ice, such as gauze, which can influence on the thermal conduction. ${ }^{30,33}$ However, the hilotherapy is more expensive, in terms of equipment and handling, and that characterizes a disadvantage over the standard cryotherapy.

This systematic review dealt with some limitations. First, there was a lack of high-quality randomized clinical trials that tested the effectiveness of the cryotherapy after surgeries of mandibular third molar. Those included in the present review had some problems, for example random generation, standardized methods for parameters measurements, and the availability of mean and standard deviation of pain, swelling, and trismus values, which support the need for more structured studies in this field. The moment of the cold application was basically the same among the included studies, but one ${ }^{22}$ applied it during the surgery, which disables the chance of a comparison with the others. Further researches on the therapeutic use of cold would be improved if standard methods were used to apply the therapy and to obtain the data, such as the visual analog scale (VAS) score for pain and three-dimensional evaluation for swelling. The variation of times of follow-up was prejudicial to complete the meta-analysis, which suggests it should also be standardized or better stated.

\section{Final Comments}

In conclusion, the cryotherapy, when applied in the early postoperative period, can manage the edema in the first days after mandibular third molar removal. Regarding trismus, the therapeutic use of cold did not present any efficacy. There are insufficient available data to support the effectiveness of this therapy in reducing postoperative pain. Well-designed randomized clinical trials testing the use of cryotherapy after third molar surgeries are needed.

\section{Conflicts of Interest and Source of Funding}

There is no conflict of interest and any source of funding.

\section{References}

1 Falci SGM, de Castro CR, Santos RC, et al. Association between the presence of a partially erupted mandibular third molar and the existence of caries in the distal of the second molars. Int J Oral Maxillofac Surg 2012;41(10):1270-1274

2 Nunn ME, Fish MD, Garcia RI, et al. Retained asymptomatic third molars and risk for second molar pathology. J Dent Res 2013;92 (12):1095-1099

3 Selmani ME, Gjorgova J, Selmani ME, Shkreta M, Duci SB. Effects of lower third molar angulation and position on lower arch crowding. Int J Orthod Milwaukee 2016;27(01):45-49

4 Zandi M, Amini P, Keshavarz A. Effectiveness of cold therapy in reducing pain, trismus, and oedema after impacted mandibular third molar surgery: a randomized, self-controlled, observer-blind, split-mouth clinical trial. Int J Oral Maxillofac Surg 2016;45(01): 118-123

5 Braimah RO, Ndukwe KC, Owotade FJ, Aregbesola SB. Oral health related quality of life (OHRQoL) following third molar surgery in Sub-Saharan Africans: an observational study. Pan Afr Med J 2016;25:97

6 Colorado-Bonnin M, Valmaseda-Castellón E, Berini-Aytés L, GayEscoda C. Quality of life following lower third molar removal. Int J Oral Maxillofac Surg 2006;35(04):343-347

7 Pandurić DG, Brozović J, Susić M, Katanec D, Bego K, Kobler P. Assessing health-related quality of life outcomes after the surgical removal of a mandibular third molar. Coll Antropol 2009;33 (02):437-447

8 Steinagel MC. Cryotherapy in sport injury management. J Athl Train 1996;31(03):277

9 Greenstein G. Therapeutic efficacy of cold therapy after intraoral surgical procedures: a literature review. J Periodontol 2007;78 (05):790-800

10 Ernst E, Fialka V. Ice freezes pain? A review of the clinical effectiveness of analgesic cold therapy. J Pain Symptom Manage 1994;9(01):56-59

11 Lehmann JF, Warren CG, Scham SM. Therapeutic heat and cold. Clin Orthop Relat Res 1974;(99):207-245 
12 Curl WW, Smith BP, Marr A, Rosencrance E, Holden M, Smith TL. The effect of contusion and cryotherapy on skeletal muscle microcirculation. J Sports Med Phys Fitness 1997;37(04):279-286

13 Forouzanfar T, Sabelis A, Ausems S, Baart JA, van der Waal I. Effect of ice compression on pain after mandibular third molar surgery: a single-blind, randomized controlled trial. Int J Oral Maxillofac Surg 2008;37(09):824-830

14 Rana M, Gellrich NC, Ghassemi A, Gerressen M, Riediger D, Modabber A. Three-dimensional evaluation of postoperative swelling after third molar surgery using 2 different cooling therapy methods: a randomized observer-blind prospective study. J Oral Maxillofac Surg 2011;69(08):2092-2098

15 Ibikunle AA, Adeyemo WL. Oral health-related quality of life following third molar surgery with or without application of ice pack therapy. Oral Maxillofac Surg 2016;20(03):239-247

16 Laureano Filho JR, de Oliveira e Silva ED, Batista CI, Gouveia FM. The influence of cryotherapy on reduction of swelling, pain and trismus after third-molar extraction: a preliminary study. J Am Dent Assoc 2005;136(06):774-778, quiz 807

17 van der Westhuijzen AJ, Becker PJ, Morkel J, Roelse JA. A randomized observer blind comparison of bilateral facial ice pack therapy with no ice therapy following third molar surgery. Int J Oral Maxillofac Surg 2005;34(03):281-286

18 Sortino F, Messina G, Pulvirenti G. [Evaluation of postoperative mucosa and skin temperature after surgery for impacted third molar]. Minerva Stomatol 2003;52(7-8):393-399

19 Moher D, Liberati A, Tetzlaff J, Altman DG; PRISMA Group. Preferred reporting items for systematic reviews and meta-analyses: the PRISMA Statement. Open Med 2009;3(03):e123-e130

20 Higgins JP, Thompson SG. Quantifying heterogeneity in a metaanalysis. Stat Med 2002;21(11):1539-1558

21 Patnaik S, Acharya SS, Kumar S, et al. Effect of cold compression therapy on pain, swelling $\&$ trismus following surgical removal of impacted mandibular third molars: a clinical research. Int J Dent Med Res 2015;1(06):18-21
22 Bastian H, Søholm B, Marker P, Eckerdal A. Comparative study of pain control by cryotherapy of exposed bone following extraction of wisdom teeth. J Oral Sci 1998;40(03):109-113

23 Courage GR, Huebsch RF. Cold therapy revisited. J Am Dent Assoc 1971;83(05):1070-1073

24 Forsgren H, Heimdahl A, Johansson B, Krekmanov L. Effect of application of cold dressings on the postoperative course in oral surgery. Int J Oral Surg 1985;14(03):223-228

25 Gelesko S, Long L, Faulk J, Phillips C, Dicus C, White RP Jr. Cryotherapy and topical minocycline as adjunctive measures to control pain after third molar surgery: an exploratory study. J Oral Maxillofac Surg 2011;69(11):e324-e332

26 Canavan WH. Drains and dressing in oral surgery and exodontia. Int J Orthod 1936;22:420-422

27 Stangel L. The value of cryotherapy and thermotherapy in the relief of pain. Physiother Can 1975;27:135-139

28 Osunde OD, Adebola RA, Omeje UK. Management of inflammatory complications in third molar surgery: a review of the literature. Afr Health Sci 2011;11(03):530-537

29 Modabber A, Rana M, Ghassemi A, et al. Three-dimensional evaluation of postoperative swelling in treatment of zygomatic bone fractures using two different cooling therapy methods: a randomized, observer-blind, prospective study. Trials 2013;14:238

30 Glass GE, Waterhouse N, Shakib K. Hilotherapy for the management of perioperative pain and swelling in facial surgery: a systematic review and meta-analysis. Br J Oral Maxillofac Surg 2016;54(08):851-856

31 Ho SS, Coel MN, Kagawa R, Richardson AB. The effects of ice on blood flow and bone metabolism in knees. Am J Sports Med 1994; 22(04):537-540

32 Pérgola PE, Johnson JM, Kellogg DL Jr, Kosiba WA. Control of skin blood flow by whole body and local skin cooling in exercising humans. Am J Physiol 1996;270(1 Pt 2):H208-H215

33 Belli E, Rendine G, Mazzone N. Cold therapy in maxillofacial surgery. J Craniofac Surg 2009;20(03):878-880 